\title{
Finite energy Dirac-Born-Infeld monopoles and string junctions
}

\author{
J. P. Gauntlett, ${ }^{1}$ C. Koehl, ${ }^{1}$ D. Mateos, ${ }^{2}$ P. K. Townsend, ${ }^{3}$ and M. Zamaklar ${ }^{3}$ \\ ${ }^{1}$ Department of Physics, Queen Mary and Westfield College, University of London, Mile End Road, London E1 4NS, United Kingdom \\ ${ }^{2}$ Departament ECM, Facultat de Física, Universitat de Barcelona and Institut de Física d'Altes Energies, Diagonal 647, \\ E-08028 Barcelona, Spain \\ ${ }^{3}$ DAMTP, University of Cambridge, Silver Street, Cambridge CB3 9EW, United Kingdom
}

(Received 8 April 1999; published 9 July 1999)

\begin{abstract}
It is shown that the world volume field theory of a single D3-brane in a supergravity D3-brane background admits finite energy, and non-singular, Abelian monopoles and dyons preserving $1 / 2$ or $1 / 4$ of the $\mathcal{N}=4$ supersymmetry and saturating a Bogomol'nyi-type bound. The $1 / 4$ supersymmetric solitons provide a world volume realization of string-junction dyons. We also discuss the dual M-theory realization of the $1 / 2$ supersymmetric dyons as finite tension self-dual strings on the M5-brane, and of the 1/4 supersymmetric dyons as their intersections. [S0556-2821(99)04116-8]

PACS number(s): 14.80.Hv, 05.45.Yv, 11.25.-w
\end{abstract}

\section{INTRODUCTION}

The $\mathcal{N}=4$ supersymmetric Yang-Mills (SYM) theory with gauge group $S U(k)$ spontaneously broken to $U(1)^{k-1}$ has a spectrum of $1 / 2$ supersymmetric magnetic monopoles and dyons which, together with the "elementary" particles of the perturbative spectrum, fill out orbits of an $S L(2 ; Z)$ electromagnetic duality group. Each such particle has an interpretation in type IIB superstring theory as an $(m, n)$ string stretched between a pair of parallel D3-branes, chosen from among $k$ parallel D3-branes. For $k=2$ there are no other particles in the spectrum but for $k \geqslant 3$ there are additional, $1 / 4$ supersymmetric, dyons that are entirely non-perturbative in the sense that they belong to $S L(2 ; \mathbb{Z})$ orbits that contain no "elementary" particles. Although these can be found as classical solutions of the SYM field equations [1-3] they were first found as type IIB superstring configurations in which three strings of different $(m, n)$ charges, attached to three D3-branes, meet at a string junction [4]. These are points at which two type IIB strings of charges $(m, n)$ and $\left(m^{\prime}, n^{\prime}\right)$ meet to form a string of charge $\left(m+m^{\prime}, n+n^{\prime}\right)$ [5]. The minimum energy state to which the configuration relaxes is one in which three strings leaving the three D3branes meet at a planar string junction [6,7].

Actually, the effective action of the D3-branes is not a SYM theory but rather a supersymmetric non-Abelian DiracBorn-Infeld (DBI) theory. The precise nature of this theory is not known (see [8] for a recent discussion) but it has an expansion in powers of $\alpha^{\prime} \mathcal{F}$ that simplifies in certain limits; $\alpha^{\prime}$ is the inverse type IIB string tension and $\mathcal{F}$ is the (background covariant) Born-Infeld field strength. If $L$ is the minimal separation between the D3-branes then (as we shall later see explicitly) $\alpha^{\prime} \mathcal{F} \sim L^{2} / \alpha^{\prime}$, so the expansion parameter is actually $L^{2} / \alpha^{\prime}$. For $L \ll \sqrt{\alpha^{\prime}}$ we need keep only the quadratic terms in $\mathcal{F}$ and the action reduces to the $\mathcal{N}=4$ SYM theory (for a vacuum type IIB background). For $L \gg \sqrt{\alpha^{\prime}}$ we cannot truncate the expansion but we may neglect the non-Abelian interactions; the action then reduces to a sum of Abelian DBI actions governing the dynamics of independent parallel D3branes. The D3-brane action depends on the supergravity background. For example, $\mathcal{F}=F-B$ where $F$ is the usual 2 -form $U(1)$ field strength, satisfying $d F=0$, and $B$ is the pullback of the background Neveu-Schwarz-NeveuSchwarz (NS-NS) 2-form potential. The D3-brane couples to the background Ramond-Ramond (R-R) gauge fields through a Wess-Zumino Lagrangian $L_{W Z}$. Let $\xi^{i}$ be the world volume coordinates, $(i=0,1,2,3)$. Omitting fermions and setting $\alpha^{\prime}=1$, the Lagrangian is then

$$
L=-e^{-\phi} \sqrt{-\operatorname{det}\left(g_{i j}+\mathcal{F}_{i j}\right)}+L_{W Z}
$$

where $g_{i j}$ is the induced world volume metric and $\phi$ the background dilaton field. For the backgrounds we consider, $\phi$ is a constant, $B$ vanishes, and $L_{W Z}$ is just the minimal coupling of the D3-brane to the 4-form gauge potential of type IIB supergravity.

For widely separated branes it makes sense to ask what a dyon looks like locally on one of the D3-branes, i.e., as a solution of the Abelian $\mathcal{N}=4$ supersymmetric DBI theory with the above Lagrangian. Because Abelian monopoles and dyons have infinite energy, this question would not make sense in the context of a $U(1)$ SYM theory, but the infinite energy has a natural interpretation in the DBI context as the energy associated with an infinite string of fixed tension. In fact, the Abelian DBI theory does have infinite energy $1 / 2$ supersymmetric solutions that appear as "spikes" on the world volume with uniform energy per unit length $[9,10]$. The "spike" solutions of the Abelian DBI theory were called bions in [7], following a slightly different use of this term in [10]. In the case of the D3-brane there are dyonic $(m, n)$-bions corresponding to infinite $(m, n)$ strings that end on the D3-brane. Although the infinite energy of a bion has a clear physical interpretation, it is nevertheless a cause for concern because, for example, solutions with infinite energy make no contribution to the semi-classical evaluation of the path-integral. One should really think of these solutions as limiting cases of the more physical situation in which the string eventually ends on another D3-brane, but it might then appear that we are forced to return to the non-Abelian DBI theory. One purpose of this paper is to show that this problem can be circumvented by replacing the second D3-brane 
by a supergravity D3-brane background. Our work therefore has links to some studies of branes in the $\operatorname{AdS}_{5} \times S^{5}$ background found by considering the near-horizon limit of the D3-brane solution, e.g. [11]. We should also point out that the infinite energy problem is circumvented by certain nonsupersymmetric solutions of the DBI action [10], although these do not correspond to Bogomol'nyi-PrasadSommerfield (BPS) states of $\mathcal{N}=4$ SYM theory.

The super D3-brane action can be consistently formulated in any background that solves the equations of type IIB supergravity. One such solution is the "supergravity D3brane." For this solution the dilaton is constant and we shall set it to zero; this corresponds to unit string coupling constant. The remaining non-vanishing fields are the metric and the 4-form potential $C$ with self-dual 5-form field strength $R=d C$. These are given by

$$
\begin{gathered}
d s^{2}=H^{-1 / 2} d s^{2}\left(\mathbb{E}^{(1,3)}\right)+H^{1 / 2} d s^{2}\left(\mathbb{E}^{6}\right) \\
R=\operatorname{vol}\left(\mathbb{E}^{(1,3)}\right) \wedge d H^{-1}+\star_{6} d H
\end{gathered}
$$

where $\star_{6}$ is the Hodge dual on $\mathbb{E}^{6}$ and $H$ is harmonic on this space. Point singularities of $H$ are coordinate singularities of the spacetime metric at connected components of a degenerate event horizon. The proper distance to the horizon on spacelike hypersurfaces of constant $\mathbb{E}^{(1,3)}$ coordinates is infinite, so that there are "internal" asymptotic infinities. If we wish the D3-brane horizon to have a single connected component then we must choose a "single-center" metric with

$$
H=1+\frac{Q}{\left|\vec{X}-\vec{X}_{0}\right|^{4}},
$$

where $\vec{X}$ are Cartesian coordinates on $\mathbb{E}^{6}$ and $\vec{X}_{0}$ is a constant $\mathbb{E}^{6}$ 6-vector. Let us now put a test D3-brane in this background, at $\vec{X}=0$. The DBI equations can now have solutions representing infinite $(m, n)$ strings that go into the internal asymptotic region of the background geometry. We shall show that there exist static bion solutions of this type, and that they have a finite energy, saturating a Bogomol'nyi-type bound. In fact, if $\left|\vec{X}_{0}\right|=L$ then the energy of the static bion is precisely $L$ times the tension of an $(m, n)$ string. Effectively, we have replaced the "second" D3-brane of the $S U(2)$ theory by a D3-brane background, thereby finding finite energy, and non-singular, supersymmetric monopoles and dyons in the Abelian DBI theory. Actually, it would be more accurate to consider this "brane in brane background" configuration as representing the large $k$ limit of an $S U(k)$ theory broken to $S U(k-1) \times U(1)$ with the $S U(k-1)$ theory replaced by the supergravity background.

The same logic that leads us to expect $1 / 2$ supersymmetric bions on the D3-brane also leads us to expect that it should be possible to find the $1 / 4$ supersymmetric string junctions this way. Consider first the case in which one of the three strings in a string junction configuration has shrunk to zero length. In this case we are left with a configuration of two "overlapping" strings of different $(m, n)$ charges each stretched between a different pair of D3-branes. In the spe- cial case of an orthogonal overlap of an F-string, charge $(1,0)$, with a D-string, charge $(0,1)$, this configuration can be represented by the array

$$
\begin{array}{llllllllll}
D 3: & 1 & 2 & 3 & - & - & - & - & - & - \\
D 1: & - & - & - & 4 & - & - & - & - & - \\
F 1: & - & - & - & - & 5 & - & - & - & -
\end{array}
$$

The corresponding $1 / 4$ supersymmetric dyon solution on the D3-brane was recently found [12]; it depends on two independent world space functions that are harmonic in the $\mathrm{Eu}-$ clidean metric. Here we shall find the general "twoharmonic-function" solution and explain its interpretation as a string junction. In a flat background these solutions again have infinite energy. The strategy explained above to find finite energy solutions can be used here too, but in this case we must use a background harmonic function $H$ with two isolated singularities; i.e., we replace Eq. (3) by

$$
H=1+\frac{Q_{1}}{\left|\vec{X}-\vec{X}_{1}\right|^{4}}+\frac{Q_{2}}{\left|\vec{X}-\vec{X}_{2}\right|^{4}}
$$

where $\vec{X}_{1}$ and $\vec{X}_{2}$ are two 6-vectors giving the positions of the background supergravity D3-branes. A string leaving the test D3-brane can now split, at a string junction, into two strings, each of which continues indefinitely into one of the two "internal" asymptotic regions of the background geometry. As we shall see, such configurations correspond to finite energy Abelian DBI solitons saturating precisely the Bogomol'nyi-type bound expected of a 1/4 supersymmetric dyon.

The 1/2 supersymmetric dyons on a D3-brane of type IIB superstring theory have an M-theory counterpart as self-dual string solitons on an M5-brane [13,7]. In flat $D=11$ spacetime these strings have infinite tension, as expected from their spacetime interpretation as semi-infinite M2-branes with a boundary on an M5-brane. However the M5-brane action can be consistently formulated in any background that solves the equations of $\mathrm{D}=11$ supergravity. One such solution is the supergravity M5-brane. The 11-metric and 4-form field strength of this solution are

$$
\begin{aligned}
& d s_{11}^{2}=U^{-1 / 3} d s^{2}\left(\mathbb{E}^{(5,1)}\right)+U^{2 / 3} d \vec{X} \cdot d \vec{X} \\
& F_{(4)}=\star_{5} d U
\end{aligned}
$$

where $\vec{X}$ are Cartesian coordinates on $\mathbb{E}^{5}$ and $U$ is a harmonic function on this space. Singularities of $U$ are just horizons of the 11-metric which are at an infinite proper distance on the spacelike hypersurfaces of constant $\mathbb{E}^{(5,1)}$ coordinates. In other words, there are again "internal" asymptotic regions into which we can take an M2-brane emanating from a test M5-brane in this background. In this way we find self-dual string solitons on the M5-brane world volume with finite tension. The 1/4 supersymmetric dyons on the type IIB D3brane also have an M-theory analogue, this time as intersecting self-dual string solitons on the M5-brane. For an M5brane in flat spacetime, these were found in [14] from the 
requirement of $1 / 4$ supersymmetry; here we show that they saturate a Bogomol'nyi-type bound, although the total energy is, of course, infinite. By considering the M5-brane in a two-center M5-brane background we are able to find solutions that represent intersecting finite-tension self-dual strings.

We begin with some details of the D3-brane Hamiltonian in a supergravity D3-brane background that we need for our subsequent discussion of finite energy bions and string junctions. We then discuss the dual M-theory realization of these solitons.

\section{D3-BRANE HAMILTONIAN IN A D3-BRANE BACKGROUND}

The Hamiltonian form of the super D3-brane Lagrangian density in a general superspace background was given in [15]. Setting fermions to zero and specializing to a background of the form assumed above we have

$$
\mathcal{L}=P_{m} \dot{X}^{m}+E^{a} \dot{V}_{a}+V_{t} \mathcal{G}-s^{a} \mathcal{P}_{a}-\frac{1}{2} \mathrm{v} \mathcal{H}
$$

where $X^{m}$ are the spacetime coordinates and $P_{m}$ the 10momentum $(m=0,1, \ldots, 9)$, and $V_{a}$ is the BI 3-vector potential $(a=1,2,3)$ and $E^{a}$ its conjugate electric field 3-vector. The constraint functions associated with the constraints imposed by the Lagrange multipliers $V_{t}, s^{a}$ and $\mathrm{V}$ are

$$
\begin{aligned}
\mathcal{H} & =G^{m n}(P-\mathcal{C})_{m}(P-\mathcal{C})_{n}+E^{a} E^{b} g_{a b}+\operatorname{det}(g+F) \\
\mathcal{G} & =\partial_{a} E^{a} \\
\mathcal{P}_{a} & =(P-\mathcal{C})_{m} \partial_{a} X^{m}+E^{b} F_{b a}
\end{aligned}
$$

where $G$ is the background metric, $g$ is the induced world space metric, $F$ the magnetic field 2 -form and $\mathcal{C}_{m}$ is the coefficient of $\dot{X}^{m}$ in the Wess-Zumino (WZ) term, i.e., $L_{W Z}$ $=\dot{X}^{m} \mathcal{C}_{m}$.

In the static gauge

$$
X^{m}=\left(\xi^{i}, \vec{X}\right),
$$

where $\xi^{i}=\left(t, \boldsymbol{\sigma}^{a}\right)$ are the world volume coordinates, we have

$$
\mathcal{C}_{m}=(\mathcal{C}, 0, \vec{C}),
$$

where, for a background of the form assumed here,

$$
\mathcal{C}=H^{-1} \text {. }
$$

In addition, the constraint $\mathcal{P}_{a}=0$ implies, in static gauge, that

$$
P_{m}=\left(-\mathcal{E},-(\vec{P}-\vec{C}) \cdot \partial_{a} \vec{X}-E^{b} F_{a b}, \vec{P}\right)
$$

where $\mathcal{E}$ is the energy density. Since $\vec{P}-\vec{C}$ vanishes for static configurations we have

$$
(P-\mathcal{C})_{m}=\left(-\mathcal{E}-H^{-1},-E^{b} F_{a b}, \overrightarrow{0}\right) .
$$

If this is now used in the constraint $\mathcal{H}=0$ we can solve for $\mathcal{E}$ to get

$$
\begin{aligned}
\left(\mathcal{E}+H^{-1}\right)^{2}= & E^{c} E^{d} F_{a c} F_{b d} \delta^{a b}+H^{-1 / 2} \\
& \times\left[E^{a} E^{b} g_{a b}+\operatorname{det}(g+F)\right]
\end{aligned}
$$

where

$$
g_{a b}=H^{-1 / 2} \delta_{a b}+H^{1 / 2} \partial_{a} \vec{X} \cdot \partial_{b} \vec{X} .
$$

When $H=1$ this reduces to the result given in [7] except for a shift of the vacuum energy. To obtain precisely the result of [7] when $H=1$ one would have to take $\mathcal{C}=H^{-1}-1$; the difference is just a gauge transformation and hence without physical significance. The choice $\mathcal{C}=H^{-1}$ is convenient because it ensures that the WZ term cancels the vacuum energy of the DBI term.

\section{FINITE ENERGY BIONS}

We shall begin by choosing the harmonic function $H$ to have the form (3) with $\vec{X}_{0}=(L, 0, \ldots, 0)$. On the $X$-axis we then have

$$
H=1+\frac{Q}{(X-L)^{4}} .
$$

We shall be interested in bions that can be interpreted as strings stretched between the test D3-brane and the source D3-brane. It is obvious that the minimum energy configuration must then be one for which the only non-zero world volume field is $X(\boldsymbol{\sigma})$ so we now set the others to zero. In this case

$$
g_{a b}=H^{-12} \delta_{a b}+H^{1 / 2} \partial_{a} X \partial_{b} X
$$

and

$$
\operatorname{det}(g+F)=H^{-3 / 2}\left[1+H|\nabla X|^{2}+H|\mathbf{B}|^{2}\right]+H^{1 / 2}(\nabla X \cdot \mathbf{B})^{2}
$$

where $\mathbf{B}$ is the magnetic field 3-vector defined by

$$
F_{a b}=\varepsilon_{a b c} B_{c} .
$$

This leads to the formula

$$
\begin{aligned}
\left(\mathcal{E}+H^{-1}\right)^{2}= & H^{-2}+H^{-1}\left[|\nabla X|^{2}+|\mathbf{E}|^{2}+|\mathbf{B}|^{2}\right]+(\nabla X \cdot \mathbf{E})^{2} \\
& +(\nabla X \cdot \mathbf{B})^{2}+|\mathbf{E} \times \mathbf{B}|^{2}
\end{aligned}
$$

which we can rewrite, for arbitrary angle $\vartheta$, as

$$
\begin{aligned}
\left(\mathcal{E}+H^{-1}\right)^{2}= & {\left[H^{-1}+\cos \vartheta(\mathbf{E} \cdot \nabla X)+\sin \vartheta(\mathbf{B} \cdot \nabla X)\right]^{2} } \\
& +H^{-1}|\mathbf{E}-\cos \vartheta \nabla X|^{2}+H^{-1}|\mathbf{B}-\sin \vartheta \nabla X|^{2} \\
& +|\sin \vartheta(\mathbf{E} \cdot \nabla X)-\cos \vartheta(\mathbf{B} \cdot \nabla X)|^{2}+|\mathbf{E} \times \mathbf{B}|^{2} .
\end{aligned}
$$

From this expression we deduce the bound 


$$
\left(\mathcal{E}+H^{-1}\right)^{2} \geqslant\left[H^{-1}+\cos \vartheta(\mathbf{E} \cdot \nabla X)+\sin \vartheta(\mathbf{B} \cdot \nabla X]^{2}\right.
$$

and hence that

$$
\mathcal{E} \geqslant \cos \vartheta(\mathbf{E} \cdot \nabla X)+\sin \vartheta(\mathbf{B} \cdot \nabla X)
$$

for any $\vartheta$. Integrating over the world space and then maximizing the right hand side with respect to $\vartheta$ we arrive at the bound

$$
M \equiv \int d^{3} \sigma \mathcal{E} \geqslant \sqrt{Q_{e}^{2}+Q_{m}^{2}}
$$

where

$$
\begin{array}{r}
Q_{e}=\int d^{3} \sigma \mathbf{E} \cdot \nabla X=\oint d \mathbf{S} \cdot \mathbf{E} X, \\
Q_{m}=\int d^{3} \sigma \mathbf{B} \cdot \nabla X=\oint d \mathbf{S} \cdot \mathbf{B} X .
\end{array}
$$

Here we have used the fact that $\mathbf{B}$ is divergence-free by definition and that $\mathbf{E}$ is divergence-free as a result of the Gauss law constraint. The final surface integrals must be taken over all components of the boundary surface of world space.

The bound (23) is saturated by solutions of

$$
\mathbf{E}=\cos \vartheta \nabla X, \quad \mathbf{B}=\sin \vartheta \nabla X,
$$

where $\tan \vartheta=Q_{m} / Q_{e}$. These are just the flat space Abelian Bogomol'nyi equations. Since both $\mathbf{E}$ and $\mathbf{B}$ are divergencefree we deduce, as in the flat-space case, that $X$ is harmonic on worldspace (in the Euclidean metric). For a single bion of charge $q$ with $X$ vanishing at spatial infinity on the brane we have

$$
X=q / 4 \pi r \quad\left(r>r_{0} \equiv q / 4 \pi L\right)
$$

where $r=|\boldsymbol{\sigma}|$ is the distance from the origin in $\mathbb{E}^{3}$.

The lower bound on $r$ comes about because the world space metric is

$$
d s_{3}^{2}=\left[H^{-1 / 2}+\left(\frac{L r_{0}}{r^{2}}\right)^{2} H^{1 / 2}\right] d r^{2}+H^{-1 / 2} d \Omega_{2}^{2},
$$

where now

$$
H=1+\frac{Q r^{4}}{L^{4}\left(r-r_{0}\right)^{4}} .
$$

As $r$ approaches $r_{0}$ from above we have

$$
d s_{3}^{2} \sim Q^{1 / 2}\left(\frac{d u}{u}\right)^{2}+Q^{-1 / 2} L^{2} u^{2} d \Omega_{2}^{2}
$$

where $u=r-r_{0}$. The proper distance to $r=r_{0}$ is therefore infinite. In fact, the sphere at $r=r_{0}$ is mapped to a single point $\vec{X}=\vec{X}_{0}$ in the transverse space. The world space of a single bion along the $\mathrm{X}$-axis therefore has two boundaries: one at $r=\infty$, where $X=0$, and another at $r=r_{0}$, where $X$ $=L$. The surface integrals of Eq. (24) vanish at the $r=\infty$ boundary, and since $X=L$ on the other boundary we have

$$
Q_{e}=L q_{e}, \quad Q_{m}=L q_{m},
$$

where

$$
\begin{aligned}
& q_{e}=\oint d \mathbf{S} \cdot \mathbf{E}=\cos \vartheta q, \\
& q_{m}=\oint d \mathbf{S} \cdot \mathbf{B}=\sin \vartheta q
\end{aligned}
$$

are the electric and magnetic charges coupling to the BI field on the brane. The bion mass is therefore

$$
M=|q| L /\left(\alpha^{\prime}\right)^{2}
$$

where we have now reinstated $\alpha^{\prime}$. This is the mass of a string of tension $|q| /\left(\alpha^{\prime}\right)^{2}$ and length $L$. Taking into acount the quantization condition on the type IIB string charges, and the fact that we have set the string coupling constant to unity, the tension of an $(m, n)$ string is $\sqrt{m^{2}+n^{2}} / \alpha^{\prime}$, so

$$
q=\alpha^{\prime} \sqrt{m^{2}+n^{2}}
$$

Reinstating $\alpha^{\prime}$ in (25) we see that the maximum value of $\alpha^{\prime} E$ or $\alpha^{\prime} B$ is $L^{2} / q \sim L^{2} / \alpha^{\prime}$, so the expansion parameter of the DBI action is $L^{2} / \alpha^{\prime}$, as claimed earlier. Actually, if $m$ or $n$ is very large then the effective expansion parameter is really smaller than $L^{2} / \alpha^{\prime}$ by a factor of $1 / \sqrt{m^{2}+n^{2}}$. For given $L$, the neglect of the DBI corrections to the SYM theory can therefore be justified by considering a sufficiently large charge, so the SYM theory is adequate for a description of macroscopic objects. The DBI corrections are important only for the description of microscopic objects.

In terms of the electrostatic analogy, the above construction can be viewed as a regularization of the infinite selfenergy of a point particle in which a point charge is replaced by a perfectly conducting charged spherical shell. This is clearly unsatisfactory as a solution to the electrostatic selfenergy problem of electrodynamics because any surface of spherical topology carrying the same total charge would serve the same purpose. Here too we could replace the spherical shell by a shell of any other shape, but in our case this has no effect on the physics. To see this we first note that the surface of the shell is, by hypothesis, an equipotential with potential $X=L$. It follows that every point on it is mapped to the point $\vec{X}=\vec{X}_{0}$ in transverse space. This point is at infinite proper distance in the transverse space metric and hence in the induced world space metric. The equipotential surface $X=L$ in $\mathbb{E}^{3}$ is therefore a point at infinity in the induced world space metric. Neighboring equipotential surfaces of constant $X<L$ can be used to define coordinates in the neighborhood of $X=L$ for which $X$ is again given by Eq. (26). It then follows that the induced world volume metric in this neigborhood is Eq. (29). But the minimum energy metric 
is determined by its behavior near points at infinity. The initial shape of the shell is therefore irrelevant to the final solution.

The spherical symmetry of the one bion solution is therefore in no way essential to the construction of finite energy bions, and multi-bion solutions can be constructed analogously: we remove $n$ closed surfaces of spherical topology from $\mathbb{E}^{3}$ and choose their potential $X$ to correspond to the center of the background D3-brane spacetime metric. The potential is set to zero at infinity. There is now a unique solution of Laplace's equation for $X$; this is the sought multibion solution. Using this solution we may compute the world space metric to which it corresponds. This metric will have $n$ points at infinity, near each of which it will take the form (29). Different shapes of the initial surfaces just correspond to different choices of coordinates for the (unphysical) Euclidean 3-space.

\section{DBI STRING JUNCTIONS}

In this section we will first derive the general bound saturated by $1 / 4$ supersymmetric dyons in a general two-center D3-brane background; then we will move on to discuss their interpretation as string junctions in the simpler case of a flat background, to return finally to their interpretation in the general case.

\section{A. The BPS bound}

So far we have considered a test D3-brane in a singlecenter parallel D3-brane background. We now want to consider a two-center background, e.g.

$$
H=1+\frac{1}{\left|\vec{X}-\vec{X}_{1}\right|}+\frac{1}{\left|\vec{X}-\vec{X}_{2}\right|}
$$

where $\vec{X}_{1}$ and $\vec{X}_{2}$ are two non-zero vectors. We may choose them to lie in the plane for which $\vec{X}=(X, Y, 0, \ldots, 0)$. A static D3-brane configuration in such a background will generally have non-constant $X(\boldsymbol{\sigma})$ and $Y(\boldsymbol{\sigma})$. The same reasoning as before now leads to the following expression for the energy density:

$$
\begin{aligned}
\left(\mathcal{E}+H^{-1}\right)^{2}= & H^{-2}+H^{-1}\left[|\nabla X|^{2}+|\nabla Y|^{2}+|\mathbf{E}|^{2}+|\mathbf{B}|^{2}\right] \\
& +(\nabla X \cdot \mathbf{E})^{2}+(\nabla Y \cdot \mathbf{E})^{2}+(\nabla X \cdot \mathbf{B})^{2}+(\nabla Y \cdot \mathbf{B})^{2} \\
& +|\mathbf{E} \times \mathbf{B}|^{2}+|\nabla X \times \nabla Y|^{2} .
\end{aligned}
$$

We can rewrite the right hand side, for arbitrary angle $\alpha$ as

$$
\begin{aligned}
& {\left[H^{-1}+\cos \alpha \mathbf{E} \cdot \nabla X-\sin \alpha \mathbf{E} \cdot \nabla Y+\sin \alpha \mathbf{B} \cdot \nabla X\right.} \\
& \quad+\cos \alpha \mathbf{B} \cdot \nabla Y]^{2}+H^{-1}[\mathbf{E}-\cos \alpha \nabla X+\sin \alpha \nabla Y]^{2} \\
& \quad+H^{-1}[\mathbf{B}-\sin \alpha \nabla X-\cos \alpha \nabla Y]^{2} n+[\sin \alpha \mathbf{E} \cdot \nabla X \\
& \quad+\cos \alpha \mathbf{E} \cdot \nabla Y-\cos \alpha \mathbf{B} \cdot \nabla X+\sin \alpha \mathbf{B} \cdot \nabla Y]^{2} \\
& \quad+|\mathbf{E} \times \mathbf{B}-\nabla X \times \nabla Y|^{2} .
\end{aligned}
$$

We thereby deduce that
$\mathcal{E} \geqslant \cos \alpha \mathbf{E} \cdot \nabla X-\sin \alpha \mathbf{E} \cdot \nabla Y+\sin \alpha \mathbf{B} \cdot \nabla X+\cos \alpha \mathbf{B} \cdot \nabla Y$,

for any $\alpha$. By integrating over the world space and maximizing the right hand side with respect to $\alpha$, we deduce that the total mass $M$ satisfies the bound

$$
M \geqslant \sqrt{\left(Q_{m}^{X}-Q_{e}^{Y}\right)^{2}+\left(Q_{m}^{Y}+Q_{e}^{X}\right)^{2}}
$$

where $\left(Q_{e}^{X}, Q_{e}^{Y}\right)$ and $\left(Q_{m}^{X}, Q_{m}^{Y}\right)$ are the non-vanishing components of the electric and magnetic charge 6-vectors

$$
\vec{Q}_{e}=\oint d \mathbf{S} \cdot \mathbf{E} \vec{X}, \quad \vec{Q}_{m}=\oint d \mathbf{S} \cdot \mathbf{B} \vec{X} .
$$

The bound is saturated when

$$
\begin{aligned}
& \mathbf{E}=\cos \alpha \boldsymbol{\nabla} X-\sin \alpha \boldsymbol{\nabla} Y, \\
& \mathbf{B}=\sin \alpha \boldsymbol{\nabla} X+\cos \alpha \boldsymbol{\nabla} Y,
\end{aligned}
$$

where

$$
\tan \alpha=\frac{Q_{m}^{X}-Q_{e}^{Y}}{Q_{e}^{X}+Q_{m}^{Y}}
$$

The mass $M$ of configurations saturating the bound may be rewritten in the form

$$
M \geqslant \sqrt{\left|\vec{Q}_{e}\right|^{2}+\left|\vec{Q}_{m}\right|^{2}+2\left|\vec{Q}_{e}\right|\left|\vec{Q}_{m}\right| \sin \xi}
$$

where $\xi$ is the angle between the two 6-vectors $\vec{Q}_{e}$ and $\vec{Q}_{m}$. This is precisely the mass formula for $1 / 4$ supersymmetric dyons in $\mathcal{N}=4 \quad D=4 \mathrm{SYM}$ theories [16,4]. It is invariant under $S O(6)$ rotations of the 6-vectors $\vec{Q}_{e}$ and $\vec{Q}_{m}$, and under an $S O(2)$ rotation of these two 6-vectors into each other, as expected from the $U(4)$ automorphism group of the $\mathcal{N}=4 \quad D=4$ supersymmetry algebra. When $\sin \xi=0$ we recover the formula for $1 / 2$ supersymmetric configurations for which $M^{2}$ is proportional to the unique quadratic $U(4)$ invariant polynomial that can be constructed from $\vec{Q}_{e}$ and $\vec{Q}_{m}$ [17].

These results actually follow directly from the supersymmetry algebra, as we now show. The $\mathcal{N}=4$ supersymmetry charges can be taken to be four two-component complex spinors of $S L(2 ; \mathbb{C})$ in the fundamental 4 representation of $U(4)$. Let $Q_{\alpha}^{i}(\alpha=1,2, i=1,2,3,4)$ be these charges, with $Q_{\alpha i}$ their complex conjugates in the $\overline{\mathbf{4}}$ representation of $U(4)$. The matrix of anticommutators of these charges is

$$
\{Q, Q\}=\left(\begin{array}{cc}
\varepsilon_{\alpha \beta} Z^{i j} & \delta_{k}^{i} P_{\alpha \dot{\alpha}} \\
\delta_{j}^{l} P_{\beta \dot{\beta}} & \varepsilon_{\dot{\alpha} \dot{\beta}} \bar{Z}_{k l}
\end{array}\right)
$$

where $P$ is the 4-momentum and $Z$ a complex central charge in the 6 representation of $S U(4)$. From the fact that the left-hand side of Eq. (43) is positive semi-definite we deduce (by considering its determinant) the bound

$$
M^{4}-2 a M^{2}+a^{2}-4 b \geqslant 0
$$


where $M^{2}=-P^{2}$, and

$$
a=\frac{1}{4} Z^{i j} \bar{Z}_{i j}, \quad a^{2}-4 b=\left|\operatorname{Pf} Z^{i j}\right|^{2}
$$

are $U(4)$-invariant polynomials [Pf denotes the Pfaffian of the antisymmetric matrix $Z$; because this is complex one must take its modulus squared to get a polynomial $U(4)$ invariant]. The bound (44) is saturated when

$$
M^{2}=a+2 \sqrt{b}
$$

where we take the positive square root because this yields the strongest bound. Comparison with Eq. (42) shows that

$$
\begin{aligned}
& a=\left|\vec{Q}_{e}\right|^{2}+\left|\vec{Q}_{m}\right|^{2}, \\
& b=\left|\vec{Q}_{e}\right|^{2}\left|\vec{Q}_{m}\right|^{2}-\left(\vec{Q}_{e} \cdot \vec{Q}_{m}\right)^{2} .
\end{aligned}
$$

When $b=0$ this reduces to the formula $M^{2}=\left|\vec{Q}_{e}\right|^{2}+\left|\vec{Q}_{m}\right|^{2}$ applicable to $1 / 2$ supersymmetric dyons. Otherwise only $1 / 4$ supersymmetry is preserved.

\section{B. The flat background case}

We shall begin our discussion of the 1/4 supersymmetric dyons, and of their interpretation as string junctions, by considering first the simpler case of a flat supergravity background, i.e., we set $H=1$. Since both $\mathbf{E}$ and $\mathbf{B}$ are divergence-free, it follows from Eq. (40) that both $X$ and $Y$ are harmonic functions, vanishing at world space infinity. As we will see, the most general string junction can be realized by choosing $X$ and $Y$ to have two centers. Hence, we choose two points $\boldsymbol{\sigma}_{1}$ and $\boldsymbol{\sigma}_{2}$ in $\mathbb{E}^{3}$ and solve the Laplace equations for $X$ and $Y$ everywhere else in $\mathbb{E}^{3}$ by setting

$$
\begin{aligned}
& X=\frac{q_{1}^{X}}{4 \pi\left|\boldsymbol{\sigma}-\boldsymbol{\sigma}_{1}\right|}+\frac{q_{2}^{X}}{4 \pi\left|\boldsymbol{\sigma}-\boldsymbol{\sigma}_{2}\right|} \\
& Y=\frac{q_{1}^{Y}}{4 \pi\left|\boldsymbol{\sigma}-\boldsymbol{\sigma}_{1}\right|}+\frac{q_{2}^{Y}}{4 \pi\left|\boldsymbol{\sigma}-\boldsymbol{\sigma}_{2}\right|}
\end{aligned}
$$

where $q_{1}^{X}, q_{2}^{X}, q_{1}^{Y}$ and $q_{2}^{Y}$ are constants. The three strings in the string junction now arise from the behavior of the solution (48) in each of the three regions of world space where it simplifies, namely near one of the singularities or far away from both of them.

Near $\boldsymbol{\sigma}=\boldsymbol{\sigma}_{1}$ we have

$$
X \sim \frac{q_{1}^{X}}{4 \pi\left|\boldsymbol{\sigma}-\boldsymbol{\sigma}_{1}\right|}+X_{0}, \quad Y \sim \frac{q_{1}^{Y}}{4 \pi\left|\boldsymbol{\sigma}-\boldsymbol{\sigma}_{1}\right|}+Y_{0},
$$

where $X_{0}$ and $Y_{0}$ are constants. In new coordinates rotated by an angle $\beta$

$$
\left(\begin{array}{l}
X^{\prime} \\
Y^{\prime}
\end{array}\right)=\left(\begin{array}{cc}
\cos \beta & \sin \beta \\
-\sin \beta & \cos \beta
\end{array}\right)\left(\begin{array}{l}
X \\
Y
\end{array}\right),
$$

with

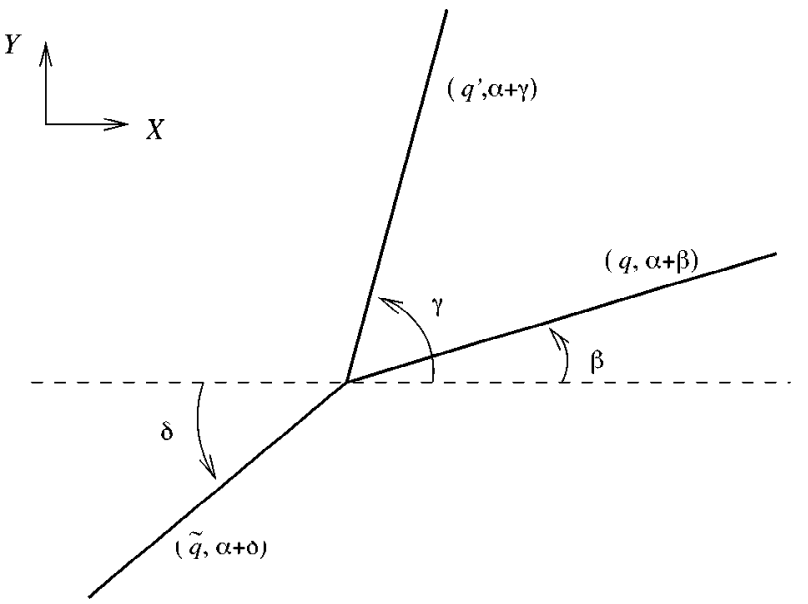

FIG. 1. The string junction described in the text.

$$
\tan \beta=q_{1}^{Y} / q_{1}^{X}
$$

we have

$$
X^{\prime} \sim \frac{q}{4 \pi\left|\boldsymbol{\sigma}-\boldsymbol{\sigma}_{1}\right|}+X_{0}^{\prime}, \quad Y^{\prime} \sim Y_{0}^{\prime}
$$

where

$$
q=\sqrt{\left(q_{1}^{X}\right)^{2}+\left(q_{1}^{Y}\right)^{2}}
$$

The electric and magnetic fields are

$$
E \sim \cos (\alpha+\beta) \nabla X^{\prime}, \quad B \sim \sin (\alpha+\beta) \nabla X^{\prime} .
$$

This looks like a $1 / 2$ supersymmetric bion with a spike along the $X^{\prime}$-axis (see Fig. 1), total charge $q$ and $\vartheta=\alpha+\beta$.

In other words, it corresponds to an $(m, n)$ string with

$$
m=q \cos (\alpha+\beta), \quad n=q \sin (\alpha+\beta) .
$$

Similarly, near $\boldsymbol{\sigma}=\boldsymbol{\sigma}_{2}$ we have

$$
X \sim \frac{q_{2}^{X}}{4 \pi\left|\boldsymbol{\sigma}-\boldsymbol{\sigma}_{2}\right|}+X_{0}^{\prime \prime}, \quad Y \sim \frac{q_{2}^{Y}}{4 \pi\left|\boldsymbol{\sigma}-\boldsymbol{\sigma}_{2}\right|}+Y_{0}^{\prime \prime} .
$$

for constants $X_{0}^{\prime \prime}$ and $Y_{0}^{\prime \prime}$. This again looks like a $1 / 2$ supersymmetric bion, now with its spike at an angle $\gamma$ to the $x$-axis, with total charge $q^{\prime}$ and $\vartheta=\alpha+\gamma$ (see Fig. 1), where

$$
q^{\prime}=\sqrt{\left(q_{2}^{X}\right)^{2}+\left(q_{2}^{Y}\right)^{2}}, \quad \tan \gamma=q_{2}^{Y} / q_{2}^{X} .
$$

In other words, the two approximate bion spikes near either singularity are rotated relative to each other by the same angle $\gamma-\beta$ in both space and charge space.

Finally, consider a region far from both singularities, where

$$
X \sim \frac{q_{1}^{X}+q_{2}^{X}}{4 \pi r}, \quad Y \sim \frac{q_{1}^{X}+q_{2}^{Y}}{4 \pi r}
$$

where $r$ is the distance from either singularity. We again have what looks like a $1 / 2$ supersymmetric bion, with its 
spike at an angle $\delta$ to the $x$-axis, with total charge $\tilde{q}$ and $\vartheta$ $=\alpha+\delta$ (see Fig. 1), where now

$$
\tilde{q}=\sqrt{\left(q_{1}^{X}+q_{2}^{X}\right)^{2}+\left(q_{1}^{Y}+q_{2}^{Y}\right)^{2}}
$$

and

$$
\tan \delta=\frac{q_{1}^{Y}+q_{2}^{Y}}{q_{1}^{X}+q_{2}^{X}}
$$

Note that $\delta$ is defined by this formula only modulo $\pi$. In this case we take the angle defining the orientation in charge space to be $\alpha+\delta$ even though the angle defining the direction in the $X-Y$ plane is $\alpha+\delta+\pi$. This is because we consider the string orientations to be such that this string "enters" the junction whereas the other two "leave" it.

One important fact that supports the string junction interpretation of the configuration we have described is that, as is easily checked, it satisfies both charge conservation (required for existence of a string junction [5]) and tension balance (required for it to be static and supersymmetric [6]).

\section{The two-center background}

The energy of the configuration described in the previous section is infinite, as expected for a flat background. To find finite energy solutions we return to the D3-brane background with $H$ given by Eq. (34). We set

$$
\begin{aligned}
& \vec{X}_{1}=\left(L_{1}, L_{1}^{\prime}, 0, \ldots, 0\right) \\
& \vec{X}_{2}=\left(L_{2}, L_{2}^{\prime}, 0, \ldots, 0\right),
\end{aligned}
$$

and we proceed according to the general prescription given earlier. We remove two 3-balls from $\mathbb{E}^{3}$ to create two boundaries, $S_{1}$ and $S_{2}$, that are shells with the topology of 2-spheres. We take these to be equipotentials with $(X, Y)$ $=\left(L_{1}, L_{1}^{\prime}\right)$ on $S_{1}$ and $(X, Y)=\left(L_{2}, L_{2}^{\prime}\right)$ on $S_{2}$. The potential at infinity vanishes. There is a unique solution to the Laplace equations for $X$ and $Y$ subject to these boundary conditions. Note that now the charges

$$
\begin{aligned}
& q_{i}^{X}=\oint_{S_{i}} d \mathbf{S} \cdot \nabla X, \\
& q_{i}^{Y}=\oint_{S_{i}} d \mathbf{S} \cdot \nabla Y, \quad(i=1,2)
\end{aligned}
$$

are fixed once the positions of the internal asymptotic regions of the background are specified. This was to be expected for the following reason. Because two of the strings are forced to go down each of these regions, the background determines completely the relative orientation among the three strings in the junction. The conditions of charge conservation and tension balance then fix the values of the charges.

The three strings arise again from the behavior of the solution near each of the shells and far away from both of them. Although the final result is the same as in the flat case,

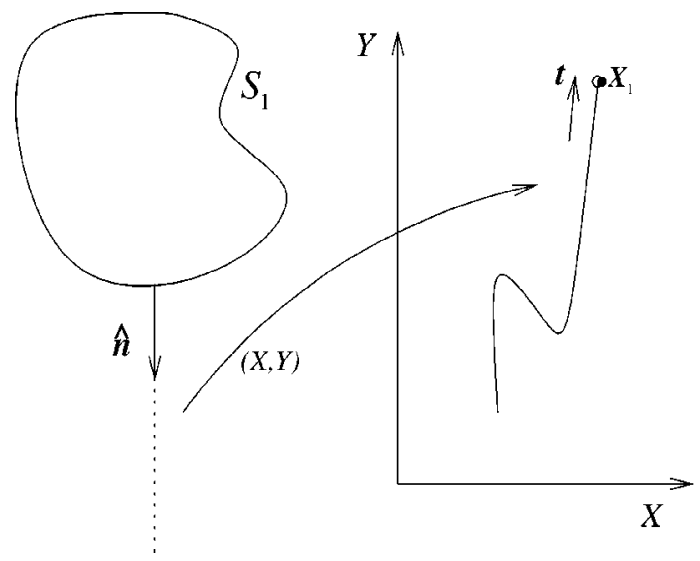

FIG. 2. The procedure described in the text to assign directions to the strings.

the analysis is not so straightforward owing to the fact that the explicit expressions for $X$ and $Y$ are no longer available. However, we will see that we have sufficient information to verify that the charge conservation and tension balance conditions are still satisfied.

The main subtlety comes about when trying to assign a direction to each of the strings. For definiteness, let us look at the region near $S_{1}$. We have $X \sim L_{1}$ and $Y \sim L_{1}^{\prime}$, so this region is mapped into one of the internal asymptotic regions of the background. We would like to determine the direction in the $X-Y$ plane along which $X$ and $Y$ approach their asymptotic values as we approach the surface $S_{1}$; this will be the direction along which we will take the string to point. We can proceed in the following way. We consider approaching $S_{1}$ along a normal to the surface, parametrized by some coordinate $r$ (see Fig. 2). This normal is mapped to a curve in the $X-Y$ plane ending on $\vec{X}_{1}$ (see Fig. 2). The tangent vector to this curve is $\vec{t}=\left(X^{\prime}, Y^{\prime}\right)$, where the prime denotes differentiation with respect to $r$. We still cannot take $\vec{t}$ to define the direction of the string, because it depends on the choice of normal to the surface along which we approach it. The most natural way to define the string direction is therefore to take the average value $\vec{T}$ of $\vec{t}$ over $S_{1}$. In other words, we define

$$
\begin{aligned}
& T_{1} \equiv \oint_{S_{1}} d S X^{\prime}=\oint_{S_{1}} d \mathbf{S} \cdot \nabla X=q_{1}^{X}, \\
& T_{2} \equiv \oint_{S_{1}} d S Y^{\prime}=\oint_{S_{1}} d \mathbf{S} \cdot \nabla Y=q_{1}^{Y},
\end{aligned}
$$

where we made use of the fact that $\nabla X$ and $\nabla Y$ are normal to $S_{1}$ (in the electrostatic analogy, this corresponds to the electric field being normal to a perfectly conducting shell). We take the string to lie at an angle $\beta$ to the $x$-axis, where

$$
\tan \beta=\frac{T_{2}}{T_{1}}=\frac{q_{1}^{Y}}{q_{1}^{X}},
$$


which is the same as Eq. (51). The electric and magnetic charges carried by this string are

$$
\begin{aligned}
& \oint_{S_{1}} d \mathbf{S} \cdot \mathbf{E}=\cos \alpha q_{1}^{X}-\sin \alpha q_{1}^{Y}=q \cos (\alpha+\beta), \\
& \oint_{S_{1}} d \mathbf{S} \cdot \mathbf{B}=\sin \alpha q_{1}^{X}+\cos \alpha q_{1}^{Y}=q \sin (\alpha+\beta),
\end{aligned}
$$

where $q$ is given in Eq. (53). The string is therefore an $(m, n)$ string with $m$ and $n$ as given in Eq. (55). We conclude that the solution near $S_{1}$ looks like a $1 / 2$ supersymmetric dyon of total charge $q$ and $\vartheta=\alpha+\beta$.

Completely analogous arguments lead to the conclusion that the solution near $S_{2}$ looks like another $1 / 2$ supersymmetric dyon, this time with its spike at an angle $\gamma$ to the $x$-axis, of total charge $q^{\prime}$ and with $\vartheta=\alpha+\gamma$, where $q^{\prime}$ and $\gamma$ are given by Eq. (57). As in the flat background case, we see that the two approximate $1 / 2$ supersymmetric bions are rotated by the same angle in space and charge space. Finally, far away from both shells the solution behaves as in Eq. (58), so it again looks like a third $1 / 2$ supersymmetric bion with spike at an angle $\delta$ to the $x$-axis, total charge $\tilde{q}$ and $\vartheta=\alpha+\delta$, with $\tilde{q}$ and $\delta$ as in Eqs. (59) and (60).

In summary, the situation is again as depicted in Fig. 1. Charge conservation and tension balance are satisfied. The difference is, of course, that now the energy is finite.

\section{FINITE ENERGY M5-BRANE STRING SOLITONS}

Infinite tension self-dual string solitons of the M5-brane world volume field theory preserving $1 / 2$ supersymmetry were found in [13], and shown to saturate a Bogomol'nyitype bound in [7]. Such solutions reduce to bions on a type IIA D4-brane, and these are T-dual to the electric D3-brane bions discussed above. Here we shall find finite tension selfdual strings on the M5-brane by considering an M5-brane in the M5-brane supergravity background (5). We shall follow the approach of [7] for which the starting point is the M5brane Hamiltonian, which we now need in an M5-brane background. This can be extracted from the Hamiltonian form of the super M5-brane in a general superspace background given in [18], by proceeding along the lines of Sec. II. In order to do this, one needs the expression for the supergravity 6-form potential $C_{(6)}$ (dual to $C_{(3)}$ ), which couples minimally to the M5-brane through the WZ term. For the M5-brane solution (5) one choice is $C_{(6)}$ $=U^{-1} \operatorname{vol}\left(\mathbb{E}^{(5,1)}\right)$; other allowed choices differ by a gauge transformation and are therefore physically equivalent. For this choice, and in the static gauge

$$
X^{m}=\left(t, \sigma^{a}, \vec{X}\right),
$$

where $\sigma^{a}(a=1, \ldots, 5)$ are the world space coordinates, the energy density for static configurations is

$$
\begin{aligned}
\left(\mathcal{E}+U^{-1}\right)^{2}= & U^{-1 / 3}\left[\operatorname{det} g+\frac{1}{2} g_{a b} g_{c d} \widetilde{\mathcal{H}}^{a c} \widetilde{\mathcal{H}}^{b d}\right. \\
& \left.+U^{1 / 3} \delta^{a b} V_{a} V_{b}\right]
\end{aligned}
$$

This is essentially the formula given in [7] except for the shift $U^{-1}$ in the energy due to the WZ term and the $U$-dependence of the right hand side, which is part explicit and part implicit in the $U$-dependence of the induced world space metric,

$$
g_{a b}=U^{-1 / 3} \delta_{a b}+U^{2 / 3} \partial_{a} \vec{X} \cdot \partial_{b} \vec{X} .
$$

Here, as in [7],

$$
\begin{aligned}
\widetilde{\mathcal{H}}^{a b} & =\frac{1}{6} \varepsilon^{a b c d e} H_{c d e} \\
V_{f} & =\frac{1}{24} \varepsilon^{a b c d e} H_{a b c} H_{d e f}
\end{aligned}
$$

where $H$ is a closed world volume 3 -form.

Our aim is now to find finite tension world volume selfdual string solitons representing an M2-brane stretched between the parallel test M5-brane and the background M5brane. We therefore choose a single-center harmonic function $U$, with a singularity on the $x$-axis at a distance $L$ from the origin in $\mathbb{E}^{5}$. Thus, on the $x$-axis we have

$$
U=1+\frac{Q}{(L-X)^{3}} .
$$

By arguments analogous to those of previous sections, the energy will be minimized when all scalars but $X$ are zero. Setting these scalars to zero, the expression for the energy then simplifies to

$$
\left(\mathcal{E}+U^{-1}\right)^{2}=U^{-2}+U^{-1}\left(|\partial X|^{2}+\frac{1}{2}|\widetilde{\mathcal{H}}|^{2}\right)+|\widetilde{\mathcal{H}} \cdot \nabla X|^{2}+|V|^{2}
$$

where

$$
\begin{aligned}
& |\widetilde{\mathcal{H}}|^{2}=\widetilde{\mathcal{H}}^{a b} \tilde{\mathcal{H}}^{c d} \delta_{a c} \delta_{b d} \\
& |V|^{2}=V_{a} V_{b} \delta^{a b}, \\
& |\partial X|^{2}=\partial_{a} X \partial_{b} X \delta^{a b} .
\end{aligned}
$$$$
|\widetilde{\mathcal{H}} \cdot \partial X|^{2}=\widetilde{\mathcal{H}}^{a b} \widetilde{\mathcal{H}}^{c d} \partial_{b} X \partial_{d} X \delta_{a c},
$$

This can be rewritten as 


$$
\begin{aligned}
\left(\mathcal{E}+U^{-1}\right)^{2}= & \left|U^{-1} \zeta^{a} \pm \widetilde{\mathcal{H}}^{a b} \partial_{b} X\right|^{2} \\
& +2 U^{-1}\left|\partial_{[a} X \zeta_{b]} \pm \frac{1}{2} \delta_{a c} \delta_{b d} \widetilde{\mathcal{H}}^{c d}\right|^{2} \\
& +U^{-1}\left(\zeta^{a} \partial_{a} X\right)^{2}+|V|^{2},
\end{aligned}
$$

where $\zeta$ is a unit length world space 5-vector, i.e.

$$
\zeta^{a} \zeta^{b} \delta_{a b}=1
$$

It follows that

$$
\mathcal{E} \geqslant\left|i_{\zeta 5}^{*}(d X \wedge H)\right|
$$

where ${ }^{*}{ }_{5}$ is the Hodge dual on world space, in the Euclidean metric, and $i_{\zeta}$ denotes contraction with the (constant) vector field $\zeta$. This inequality is saturated when

$$
H= \pm i_{\zeta}\left(*{ }_{5} d X\right), \quad \mathcal{L}_{\zeta} X=0
$$

where $\mathcal{L}_{\zeta}$ is the Lie derivative with respect to $\zeta$. These conditions, which are the same as those of the flat background case, imply $\mathcal{L}_{\zeta} H=0$. The minimum energy solution is therefore invariant under translations in the $\zeta$ direction, which we may take to be compact with length $l$. The total energy is then

$$
E \geqslant l \times|Z|
$$

where $Z$ is the topological charge found by integrating the 4-form $d X \wedge H$ over the 4-dimensional hypersurface $w_{\zeta}$ with normal $\zeta$, i.e.

$$
Z=\int_{w_{\zeta}} d X \wedge H=\int_{\partial w_{\zeta}} X H
$$

where the last equality follows from the fact that $d H=0$. The absolute value of $Z$ can be interpreted as the tension of the string soliton.

Because $H$ is closed, it also follows that $X$ is harmonic on $w_{\zeta}$. For a single string with $X$ vanishing at transverse infinity on the M5-brane we have

$$
X=\frac{q}{2 \pi^{2} r^{2}} \quad\left(r>r_{0} \equiv \sqrt{q / 2 \pi^{2} L}\right)
$$

where $r \equiv \sqrt{\sigma_{1}^{2}+\cdots+\sigma_{4}^{2}}$ is the distance from the origin in $w_{\zeta}$. The lower bound on $r$ arises for reasons analogous to those of Sec. III: as $r$ approaches $r_{0}$ from above, $X$ approaches $L$ and

$$
U \sim \frac{Q}{[L-X(r)]^{3}} \sim \frac{Q r_{0}^{3}}{8 L^{3} u^{3}}
$$

where $u=r-r_{0}$. The asymptotic world space metric is

$$
d s^{2} \sim C u(\zeta \cdot d x)^{2}+Q^{2 / 3}\left(\frac{d u}{u}\right)^{2}+C r_{0}^{2} u d \Omega_{3}^{2}
$$

where $C=2 L / Q^{1 / 3} r_{0}$. The proper distance to $u=0$ is infinite so we should restrict the world space coordinate $u$ to be positive. Note that not only does the 3 -sphere at constant $u$ contract to a point at $u=0$, but so also does the circle of length $l$ along the $\zeta$ direction. Thus, it is not only the 3 -sphere of radius $r_{0}$ that is mapped to the single point $\vec{X}$ $=(L, 0, \ldots, 0)$ in the target space; all points on the string core at $r=r_{0}$ are also mapped to this point.

We are now in a position to evaluate the integral of Eq. (78) for the tension of the M5-brane string soliton. There are two components of $\partial w_{\zeta}$. One is at $r=\infty$, where $X=0$, and the other is at $r=r_{0}$, where $X=L$. The only contribution to the integral comes from the latter boundary, so the string tension is

$$
Z=L q
$$

where

$$
q \equiv \int_{S^{3}} H
$$

is the string charge (as the string threads the 3-sphere). The total energy is therefore $l L q$, as one would expect for a membrane of area $l L$ and charge $q$.

\section{INTERSECTING STRINGS ON THE M5-BRANE}

We now turn to the study of two intersecting self-dual strings on the M5-brane, corresponding to the spacetime configuration

$$
\begin{array}{lllllllllll}
\text { M5: } & 1 & 2 & 3 & 4 & 5 & - & - & - & - & - \\
\text { M2: } & - & - & - & 4 & - & 6 & - & - & - & - \\
M 2: & - & - & - & - & 5 & - & 7 & - & - & -
\end{array}
$$

Therefore we allow for two scalars to be excited, in which case

$$
\begin{aligned}
\left(\mathcal{E}+U^{-1}\right)^{2}= & U^{-2}+U^{-1}\left[|\partial X|^{2}+|\partial Y|^{2}+\frac{1}{2}|\tilde{\mathcal{H}}|^{2}\right] \\
& +\frac{1}{2}|\partial X \wedge \partial Y|^{2}+|\tilde{\mathcal{H}} \cdot \partial X|^{2}+|\tilde{\mathcal{H}} \cdot \partial Y|^{2} \\
& +(\partial X \cdot \widetilde{\mathcal{H}} \cdot \partial Y)^{2}+|V|^{2}
\end{aligned}
$$

where

$$
\begin{aligned}
& \frac{1}{2}|\partial X \wedge \partial Y|^{2} \equiv|\partial X|^{2}|\partial Y|^{2}-(\partial X \cdot \partial Y)^{2}, \\
& \partial X \cdot \widetilde{\mathcal{H}} \cdot \partial Y \equiv \partial_{a} X \widetilde{\mathcal{H}}^{a b} \partial_{b} Y .
\end{aligned}
$$

We are interested in minimum energy configurations associated with two membranes that intersect the M5-brane in two non-parallel directions, specified by two constant 5-vectors. Let these vectors span the 4-5 plane and let $\sigma^{\alpha}(\alpha=1,2,3)$ be the coordinates for the orthogonal complement of world space. In this case, we have 


$$
\begin{aligned}
& V_{\alpha}=\epsilon_{\alpha \beta \gamma} \widetilde{\mathcal{H}}^{\beta 5} \widetilde{\mathcal{H}}^{\gamma 4}+\frac{1}{2} \epsilon_{\alpha \beta \gamma} \widetilde{\mathcal{H}}^{\beta \gamma} \widetilde{\mathcal{H}}^{45} \\
& V_{4}=\frac{1}{2} \epsilon_{\alpha \beta \gamma} \widetilde{\mathcal{H}}^{\alpha \beta} \tilde{\mathcal{H}}^{5 \gamma} \\
& V_{5}=-\frac{1}{2} \epsilon_{\alpha \beta \gamma} \widetilde{\mathcal{H}}^{\alpha \beta} \widetilde{\mathcal{H}}^{4 \gamma} .
\end{aligned}
$$

For notational convenience we define

$$
E^{\alpha}=\tilde{\mathcal{H}}^{\alpha 5}, \quad B^{\alpha}=\tilde{\mathcal{H}}^{\alpha 4},
$$

and

$$
K_{\alpha}=\frac{1}{2} \varepsilon_{\alpha \beta \gamma} \widetilde{\mathcal{H}}^{\beta \gamma}, \quad \Pi=\widetilde{\mathcal{H}}^{45}
$$

so that

$$
\begin{aligned}
\mathbf{V} & =\mathbf{E} \times \mathbf{B}+\Pi \mathbf{K} \\
V_{4} & =-\mathbf{E} \cdot \mathbf{K} \\
V_{5} & =\mathbf{B} \cdot \mathbf{K} .
\end{aligned}
$$

The right hand side of Eq. (84) may now be rewritten as

$$
\begin{aligned}
U^{-2} & +U^{-1}\left[|\nabla X|^{2}+|\nabla Y|^{2}+|\mathbf{E}|^{2}+|\mathbf{B}|^{2}\right]+|\nabla X \times \nabla Y|^{2}+U^{-1}\left[\Pi^{2}+\left(\partial_{4} X\right)^{2}+\left(\partial_{5} X\right)^{2}+\left(\partial_{4} Y\right)^{2}+\left(\partial_{5} Y\right)^{2}+|\mathbf{K}|^{2}\right] \\
& +\left|\partial_{4} X \nabla Y-\partial_{4} Y \nabla X\right|^{2}+\left|\partial_{5} X \nabla Y-\partial_{5} Y \nabla X\right|^{2}+\left(\partial_{4} X \partial_{5} Y-\partial_{4} Y \partial_{5} X\right)^{2}+\left(\mathbf{B} \cdot \nabla X-\Pi \partial_{5} X\right)^{2}+\left(\mathbf{E} \cdot \nabla X+\Pi \partial_{4} X\right)^{2} \\
& +\left(\mathbf{B} \cdot \nabla Y-\Pi \partial_{5} Y\right)^{2}+\left(\mathbf{E} \cdot \nabla Y+\Pi \partial_{4} Y\right)^{2}+\left|\mathbf{K} \times \nabla X+\mathbf{B} \partial_{4} X+\mathbf{E} \partial_{5} X\right|^{2}+\left|\mathbf{K} \times \nabla Y+\mathbf{B} \partial_{4} Y+\mathbf{E} \partial_{5} Y\right|^{2}+|\mathbf{E} \times \mathbf{B}+\Pi \mathbf{K}|^{2} \\
& +(\mathbf{K} \cdot \mathbf{E})^{2}+(\mathbf{K} \cdot \mathbf{B})^{2}+\left[\mathbf{K} \cdot \nabla X \times \nabla Y+\Pi\left(\partial_{4} X \partial_{5} Y-\partial_{4} Y \partial_{5} X\right)+\mathbf{B} \cdot \nabla X \partial_{4} Y-\mathbf{B} \cdot \nabla Y \partial_{4} X+\mathbf{E} \cdot \nabla X \partial_{5} Y-\mathbf{E} \cdot \nabla Y \partial_{5} X\right]^{2} .
\end{aligned}
$$

We start to see the beginning of what looks like an expression for the energy of static configurations on the 3-brane obtained by compactifying the M5-brane on a 2-torus, as expected from the standard duality between M-theory and the type IIB superstring theory. The comparison is, however, complicated by several factors. One is that the notion of "static" for an M5-brane configuration does not coincide with what we meant by this term in the D3-brane case. The point is that the 3-form $H$ on the M5-brane world space includes configuration space variables and their conjugate momenta (via a constraint relating $\widetilde{\mathcal{H}}$ to the momentum conjugate to the 2-form potential [18]). A related point is that the space transverse to the M5-brane is only 5-dimensional whereas the space transverse to the D3-brane is 6-dimensional. The "sixth" scalar, and its conjugate momentum are encoded in the 3-form $H$. In fact, one can roughly view $\mathbf{K}$ as the field strength of the sixth scalar and $\Pi$ as its conjugate momentum, although $\mathbf{K}$ is only a closed 1-form when $\mathbf{E}$ and $\mathbf{B}$ are independent of $\sigma^{4}$ and $\sigma^{5}$. Clearly, a direct comparison with the D3-brane energy would require that we include in the latter some "3-scalar"' terms as well as some terms that vanish for static configurations (in the D3-brane sense). Instead, we shall set $\mathbf{K}$ and $\Pi$ to zero and compare with the expression for the energy of static " 2 -scalar' D3-brane configurations. Note that, under these conditions, the closure of the 3-form $H$ implies that $\mathbf{E}$ and $\mathbf{B}$ are divergence-free, in which case they may be identified as the electric and magnetic fields on the D3-brane.

With this simplification, the expression (90) can be written, for arbitrary angle $\varphi$, as

$$
\begin{aligned}
{\left[U^{-1}\right.} & +\cos \varphi \mathbf{E} \cdot \nabla X-\sin \varphi \mathbf{E} \cdot \nabla Y+\sin \varphi \mathbf{B} \cdot \nabla X+\cos \varphi \mathbf{B} \cdot \nabla Y]^{2}+|\mathbf{E} \times \mathbf{B}-\nabla X \times \nabla Y|^{2}+\left(\partial_{4} X \partial_{5} Y-\partial_{4} Y \partial_{5} X\right)^{2}+U^{-1}\left[\left(\partial_{4} X\right)^{2}\right. \\
& \left.+\left(\partial_{5} X\right)^{2}+\left(\partial_{4} Y\right)^{2}+\left(\partial_{5} Y\right)^{2}\right]+\left|\partial_{4} X \nabla Y-\partial_{4} Y \nabla X\right|^{2}+\left|\partial_{5} X \nabla Y-\partial_{5} Y \nabla X\right|^{2}+\left(\partial_{4} X \partial_{5} Y-\partial_{4} Y \partial_{5} X\right)^{2} \\
& +U^{-1}|\mathbf{E}-\cos \varphi \nabla X+\sin \varphi \nabla Y|^{2}+U^{-1}|\mathbf{B}-\sin \varphi \nabla X-\cos \varphi \nabla Y|^{2}+[\sin \varphi \mathbf{E} \cdot \nabla X+\cos \varphi \mathbf{E} \cdot \nabla Y-\cos \varphi \mathbf{B} \cdot \nabla X \\
& +\sin \varphi \mathbf{B} \cdot \nabla Y]^{2}+\left|\mathbf{B} \partial_{4} X+\mathbf{E} \partial_{5} X\right|^{2}+\left|\mathbf{B} \partial_{4} Y+\mathbf{E} \partial_{5} Y\right|^{2}+\left[\mathbf{B} \cdot \nabla X \partial_{4} Y-\mathbf{B} \cdot \nabla Y \partial_{4} X+\mathbf{E} \cdot \nabla X \partial_{5} Y-\mathbf{E} \cdot \nabla Y \partial_{5} X\right]^{2} .
\end{aligned}
$$

We thereby deduce the bound

$$
\mathcal{E} \geqslant \cos \varphi \mathbf{E} \cdot \nabla X-\sin \varphi \mathbf{E} \cdot \nabla Y+\sin \varphi \mathbf{B} \cdot \nabla X+\cos \varphi \mathbf{B} \cdot \nabla Y
$$

for any $\varphi$, with equality when both

$$
\partial_{4} X=\partial_{5} X=\partial_{4} Y=\partial_{5} Y=0,
$$

and

$$
\mathbf{E}=\cos \varphi \nabla X-\sin \varphi \nabla Y
$$

$$
\mathbf{B}=\sin \varphi \nabla X+\cos \varphi \nabla Y .
$$

These conditions are precisely those found in [14] to be associated with $1 / 4$ supersymmetry. When they are satisfied, the 3-vectors $\mathbf{E}$ and $\mathbf{B}$ are independent of $\sigma^{4}$ and $\sigma^{5}$. The energy of the minimal energy configuration on the $T^{2}$-wrapped M5-brane is therefore proportional to the expression (42) for the energy of a 1/4 supersymmetric dyon on the D3-brane.

The 1/4 supersymmetric dyons discussed earlier thus aquire an M-theory interpretation as intersections on the M5- 
brane of two string boundaries of two M2-branes. When the two M2-branes intersect orthogonally, so do the strings. This maps to a type IIB configuration composed of two orthogonal bion spikes that are also othogonal in charge space. A rotation of the M2-branes away from orthogonality preserving $1 / 4$ supersymmetry corresponds to a simultaneous rotation (of one membrane relative to the other) by the same angle in the M5-brane and in the space transverse to it. This corresponds in the type IIB theory to a simultaneous rotation by the same angle in transverse space and charge space.

We can now find finite energy $1 / 4$ supersymmetric solitons on the M5-brane by the procedure described earlier, but we should mention that the relation between the $1 / 4$ supersymmetric M5-brane solitons and the string-junction dyons on the D3-brane is straightforward only in the case of the infinite energy solutions, in a flat background, because in general the harmonic function $U$ of the M5-brane background is not the same as the harmonic function $H$ of the D3-brane background. The M5-brane transverse space is only five-dimensional whereas it is six-dimensional for the D3-brane.

We should also mention that there is more than one possible interpretation of D3-brane dyons as M5-brane solitons. Recall that the 6-space transverse to the D3-brane is reduced to a 5-space transverse to the M5-brane. This leads one to wonder what the M-theory interpretation is of a D3-brane bion with its spike in this sixth dimension. If the D3-brane bion is purely magnetic then the answer is that it corresponds to a marginal bound state of the M5-brane with an M-wave travelling along it. If we compactify along the direction of the wave then we get a D0-brane on a D4-brane of type IIA superstring theory. This has a T-dual in type IIB superstring theory as a D-string ending on a D3-brane, which is a magnetic bion from the perspective of the D3-brane world volume. One gets an electric D3-brane bion from the same M-theory configuration by reducing along another direction in the M5-brane orthogonal to the M-wave. This leads to a wave on a D4-brane. T-dualizing along the wave direction then yields the desired configuration.

\section{DISCUSSION}

We have seen that finite energy configurations of type IIB superstring theory in which $(m, n)$ strings are suspended between D3-branes can be found as $1 / 2$ or $1 / 4$ supersymmetric solitons on a test D3-brane in a supergravity D3-brane background, the $1 / 4$ supersymmetric solitons having a natural interpretation in terms of string junctions. These solitons are Abelian analogues of the finite energy supersymmetric solitons of $D=4 \mathcal{N}=4$ SYM theory. There are similar $1 / 2$ and $1 / 4$ supersymmetric solitons on a test M5-brane in a supergravity M5-brane background, related by dualities to those on the D3-brane. In our exposition of these results we glossed over a few points, and we shall conclude with a brief discussion of them.

The first point has to do with whether the bion solutions we have have found are really non-singular. Consider the one bion solution in which the sphere in $\mathbb{E}^{3}$ of radius $r_{0}$ is mapped by $X(r)$ to the "center" $X=L$ of the background metric. The induced world space metric near $r=r_{0}$ was given as $d s_{3}^{2}$ in Eq. (29), with $u=r-r_{0}$. The corresponding world volume metric is, for $Q=1$ and after a constant rescaling of the time coordinate,

$$
d s_{4}^{2}=-u^{2} d t^{2}+\left(\frac{d u}{u}\right)^{2}+L^{2} u^{2} d \Omega_{2}^{2}
$$

The submanifold with $d \Omega_{2}^{2}=0$ is just $\operatorname{AdS}_{2}$, with $u=0$ a Killing horizon of $\partial_{t}$. This is at infinite proper distance on spacelike geodesics of constant $t$ but at finite affine parameter on timelike or null geodesics. This is not unexpected because the singularity of the spacetime metric at its center is also a Killing horizon at a finite affine parameter on timelike or null geodesics. However, although the singularity at $u=0$ is just a coordinate singularity of $\mathrm{AdS}_{2}$, it is a curvature singularity of the full 4-metric (95) because the 2-spheres at constant $u$ shrink to points at $u=0$.

It is not clear to us whether this is really a problem because there are no test particles on the D3-brane moving on timelike geodesics in this world volume metric. If we were to consider the time dependent problem in which a wave on the D3-brane scatters from a bion then we would have to first solve the DBI equations for this problem and then recompute the world volume metric. In general, one would expect the result to differ from the static bion metric of Eq. (95), which would be of direct relevance to the time-dependent problem only if all scalar fluctuations were to vanish. It seems unlikely that the DBI equations will have such solutions when linearized about the static bion configuration (rather than about the Minkowski vacuum) but we have not investigated this in detail. The whole area of time-dependent scattering solutions involving bions deserves a separate study.

A second point concerns the string junction interpretation of the 1/4 supersymmetric DBI dyons on the D3-brane. Recall that this involves, in the case of a flat background, singularities of two harmonic functions $X$ and $Y$ at points with $\mathbb{E}^{3}$ coordinates $\boldsymbol{\sigma}_{1}$ and $\boldsymbol{\sigma}_{2}$. Near either singularity, and far from both, the solution is approximately that of a single $1 / 2$ supersymmetric bion. The term "far from" here refers to a region in which the distance from either singularity (in the $\mathbb{E}^{3}$ metric) is much larger than $\left|\boldsymbol{\sigma}_{1}-\boldsymbol{\sigma}_{2}\right|$. The larger $\left|\boldsymbol{\sigma}_{1}-\boldsymbol{\sigma}_{2}\right|$ is, the further out is this region and the smaller is the deviaton of the worldspace metric from the Euclidean metric. Thus, for large $\left|\boldsymbol{\sigma}_{1}-\boldsymbol{\sigma}_{2}\right|$ it is more natural to interpret the world space configuration as one for which two strings meet the D3-brane at widely separated points. Only as the separation of these points decreases does the string junction interpretation become the natural one. Even in this case one could interpret the deviation of the world space from the Euclidean metric as that required to support two strings meeting the D3-brane at the points $\boldsymbol{\sigma}=\boldsymbol{\sigma}_{1}$ and $\boldsymbol{\sigma}=\boldsymbol{\sigma}_{2}$, just as the single bion solution has the alternative interpretation as the deviation required to support an attached string [10,19], rather than as the string itself.

Finally, we should mention that string junction dyons constitute a special case of string web dyons [20] in which three or more parallel D3-branes are connected by a network 
of strings meeting at string junctions [21]. The logic of our approach would suggest that these should also have a world volume interpretation but we have not been able to verify this. In the first instance one could seek new infinite energy solutions in which strings arriving from other branes at infinity are realized as point charges. The topological features of the network might then be encoded in branch cuts, but it is unclear to us whether this makes sense, and if so how it works in detail.

\section{ACKNOWLEDGMENTS}

D.M. thanks colleagues at DAMTP for the hospitality during the time this work was performed. D.M. is supported by the Comissionat per a Universitats i Recerca de la Generalitat de Catalunya. J.P.G. thanks EPSRC for financial support. We thank Fay Dowker, Gary Gibbons and George Papadopoulos for helpful conversations, and Barak Kol for raising the issue of string webs.
[1] K. Hashimoto, H. Hata, and N. Sasakura, Phys. Lett. B 431, 303 (1998); Nucl. Phys. B535, 83 (1998).

[2] T. Kawano and K. Okuyama, Phys. Lett. B 432, 338 (1998).

[3] K. Lee and P. Yi, Phys. Rev. D 58, 066005 (1998).

[4] O. Bergman, Nucl. Phys. B525, 104 (1998).

[5] J. H. Schwarz, Nucl. Phys. B (Proc. Suppl.) 55, 1 (1997).

[6] S. Mukhi and S. Dasgupta, Phys. Lett. B 423, 261 (1998); S-J. Rey and J-T. Yee, Nucl. Phys. B526, 229 (1998).

[7] J. P. Gauntlett, J. Gomis, and P. K. Townsend, J. High Energy Phys. 01, 003 (1998).

[8] M. J. Perry and D. Brecher, Nucl. Phys. B527, 121 (1998); D. Brecher, Phys. Lett. B 442, 117 (1998).

[9] C. Callan and J. Maldacena, Nucl. Phys. B513, 198 (1998).

[10] G. W. Gibbons, Nucl. Phys. B514, 603 (1998).

[11] S. J. Rey and J. Yee, "Macroscopic strings as heavy quarks in large N gauge theory and anti-de Sitter space,', hep-th/9803001.
[12] G. Papadopoulos and J. Gutowski, "The dynamics of D3brane dyons and toric hyper-Kahler geometry," hep-th/9811207.

[13] P. S. Howe, N. D. Lambert, and P. C. West, Nucl. Phys. B515, 203 (1998).

[14] J. P. Gauntlett, N. D. Lambert, and P. C. West, “Supersymmetric five-brane solitons,' hep-th/9811024.

[15] E. Bergshoeff and P. K. Townsend, Nucl. Phys. B531, 226 (1998).

[16] C. Fraser and T. J. Hollowood, Phys. Lett. B 402, 106 (1997).

[17] H. Osborn, Phys. Lett. 83B, 321 (1979).

[18] E. Bergshoeff, D. Sorokin, and P. K. Townsend, Nucl. Phys. B533, 303 (1998).

[19] A. Hashimoto, Phys. Rev. D 57, 6441 (1998).

[20] O. Bergman and B. Kol, Nucl. Phys. B536, 149 (1998).

[21] A. Sen, J. High Energy Phys. 03, 005 (1998). 